\title{
Adalimumab in the Management of Isotretinoin-Induced Acne Fulminans: Report of a Case
}

\author{
Claudio Marasca Gabriella Fabbrocini Luisa Abategiovanni Elisa Camela \\ Mariateresa Nocerino Adriana Di Guida Gaia De Fata Salvatores
}

Section of Dermatology, Department of Clinical Medicine and Surgery, University of Naples Federico II, Naples, Italy

\section{Established Facts}

- Acne fulminans (AF) is a rare and severe form of acne vulgaris which usually affects male adolescents between 13 and 22 years, with a prior history of acne vulgaris.

- Etiology is still unclear: steroid hormones and isotretinoin play a role.

- Four types of AF exist according to etiology and systemic involvement: idiopathic AF and isotretinoininduced AF, with/without systemic symptoms.

- Treatment is challenging: it should be started immediately after the diagnosis and based on the combination of steroids and isotretinoin regardless of the etiology.

\section{Novel Insights}

- Novel therapies are emerging: biologicals may represent a unique resource for refractory cases.

- TNF- $\alpha$ is a crucial cytokine involved in the pathogenesis of inflammation underlying acne fulminans (AF).

- Adalimumab, an anti-TNF-a monoclonal antibody, is approved for the treatment of psoriasis and hidradenitis suppurativa in adolescents ( $\geq 12$ years) and off-label administered in several dermatological conditions, that is pyoderma gangrenosum and AF.

\section{Keywords}

Acne fulminans $\cdot$ Isotretinoin $\cdot$ Adalimumab $\cdot$ Treatment

\section{Abstract}

Acne fulminans (AF) is a rare, acute, and severe form of acne vulgaris often associated with systemic symptoms. Its treat- ment is challenging and controversial. We report a case of isotretinoin-induced AF (IIAF) in a 12-year-old boy resistant to traditional therapies (oral steroids and isotretinoin). The patient was successfully treated with adalimumab that can be considered an effective off-label option in the treatment of resistant IIAF in children.

(c) 2021 S. Karger AG, Basel karger@karger.com www.karger.com/sad

(C) 2021 S. Karger AG, Basel

Karger ${ }^{\prime}=$
Elisa Camela

Section of Dermatology, Department of Clinical Medicine and Surgery University of Naples Federico II

Via Sergio Pansini 5, IT-80131 Naples (Italy)

elisacamela@gmail.com 
Fig. 1. On admission. a Back. b. Chest. c Face.
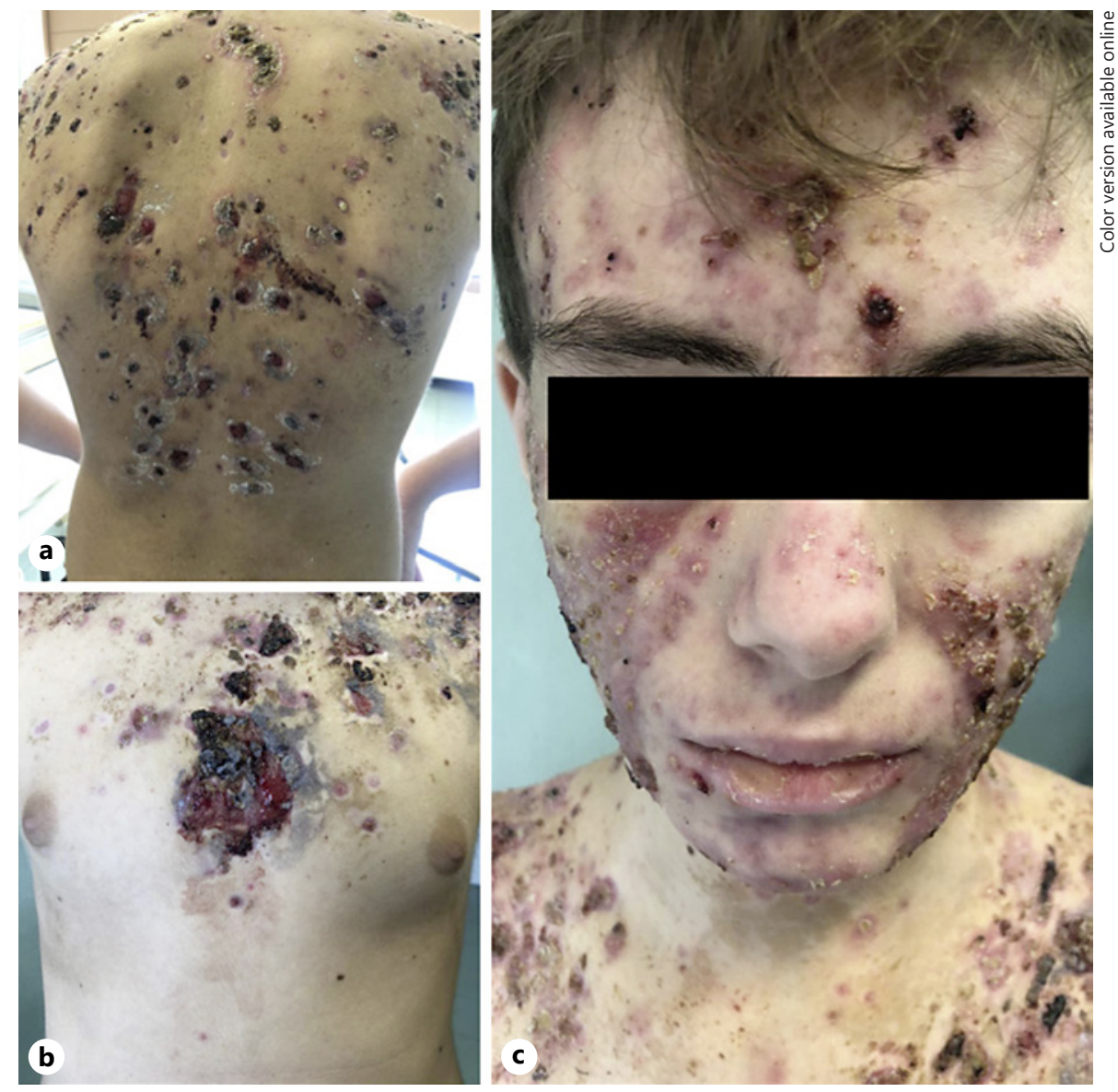

\section{Introduction}

Acne fulminans (AF) is a rare, severe form of acne vulgaris characterized by an acute onset of painful, inflammatory, ulcerative lesions covered with hemorrhagic crusts, which usually involves scarring. The lesions usually occur on the upper trunk, while the face is generally less severely involved. It may be associated with systemic symptoms (SSs) such as fever, arthralgia, malaise, loss of appetite, and abnormal laboratory findings [1]. The disorder affects more frequently young males between 13- and 22-year old with a prior history of acne [1-3]. Although the etiology of AF is not well understood, pharmacologic agents such as steroid hormones and isotretinoin have been shown to be wellknown triggers $[4,5]$. Management of AF is complex since traditional therapies are often ineffective. The treatment is based on a combination of oral steroids and isotretinoin. However, there are refractory cases that can benefit from alternative therapies including bi- ological drugs [6]. Herein, we report a case of isotretinoin-induced AF (IIAF) successfully treated with adalimumab (ADA).

\section{Case Report/Case Presentation}

A 12-year-old boy came to our clinic for an abrupt severe worsening of his papulopustular acne. He was previously treated with topical and systemic antibiotics (lymecycline - doxycycline) without any significant improvement. For this reason, he was prescribed low-dose isotretinoin $(0.2 \mathrm{mg} / \mathrm{kg} /$ day equal to $10 \mathrm{mg} /$ day $)$. Nevertheless, 2 weeks after starting treatment with isotretinoin, skin lesions progressively worsened with the development of disseminated ulcerative lesions covered with serous-hemorrhagic crusts over the face and upper trunk; some of them, the biggest, presented an overlying hypergranulation tissue. Few pustules, post-inflammatory hyperpigmentation, and scars were also seen (Fig. 1). The clinical conditions were associated with SSs such as fever, malaise, abdominal, and joint pain together with weight loss.

Routine blood test showed severe neutrophilic leukocytosis (total WBC count: $32.72 \times 10^{3} / \mu \mathrm{L}$; neutrophils: $88.9 \%, 29.09 \times 10^{3} /$ 
Fig. 2. Before starting ADA therapy. Face. ADA, adalimumab.

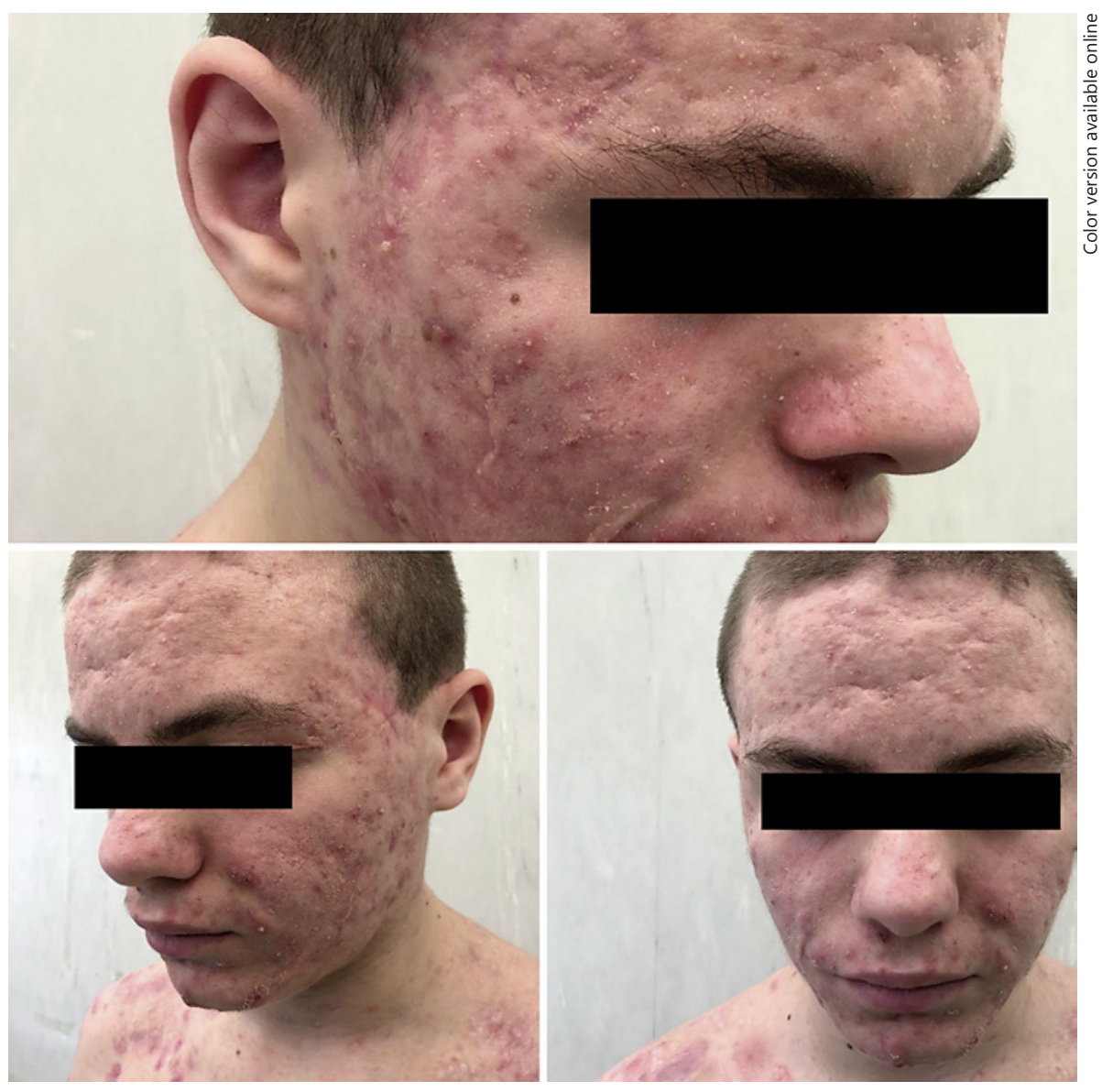

$\mu \mathrm{L}$ ) and thrombocytosis (platelet count: $749 \times 10^{3} / \mu \mathrm{L}$ ) and an increase in liver enzyme level (AST/SGOT: $40 \mathrm{U} / \mathrm{L} ; \mathrm{AST} / \mathrm{SGPT}$ : 77 $\mathrm{U} / \mathrm{L}$ ) and C-reactive protein (CRP: $7.14 \mathrm{mg} / \mathrm{dL}$ ). Based on the medical history and clinical cutaneous manifestations, a diagnosis of IIAF-SS was made.

Promptly, a systemic corticosteroid and antibiotic therapy was started as follows: prednisone was administered orally at the dose of $25 \mathrm{mg} /$ day for 2 weeks then tapered to $25 \mathrm{mg} /$ day on alternating days with $12.5 \mathrm{mg} /$ day for further 2 weeks; concomitantly, intramuscular ceftriaxone was provided at $1 \mathrm{~g} /$ day for 7 days, followed by 1 week-oral amoxicillin/clavulanic acid 875 $\mathrm{mg} / 125 \mathrm{mg} /$ day and 2 week-oral clindamycin $150 \mathrm{mg} \times 4$ /day. Topical antiseptics were applied daily. The therapy gave rapid and successful results as early as 2 weeks when most lesions healed and were replaced by scar tissue. Such scars were predominantly keloidal on the face with disfiguring large shoots of granulation tissue.

Notwithstanding, the improvement was transient. Hence, a second attempt with low-dose isotretinoin ( $10 \mathrm{mg} /$ day) on alternating days, and oral corticosteroids (prednisone $10 \mathrm{mg} /$ day) was interrupted because of acne exacerbation. A new cycle of systemic corticosteroids and antibiotics was started, with an initial improvement since new scattered papules and pustules, mainly involving the face, appeared 2 days after treatment with- drawal (Fig. 2). Likewise the first episode, they were accompanied by SSs like joint pain and malaise. Given the reoccurrence of AF, the patient was screened for biological therapy and performed routine blood tests, hepatitis markers (HBV and HCV), and Mycobacterium tuberculosis test (Quantiferon TB gold test) which were unremarkable. Hence, after the signature of the informed consent by the parents, off-label subcutaneous ADA was prescribed $(80 \mathrm{mg}$ at baseline followed by $40 \mathrm{mg}$ every 2 weeks). Notably, inflammatory lesions resolved and scars dramatically improved as early as 3 weeks; in addition, at 3-month follow-up, the patient is still in clinical remission without flares (Fig. 3).

\section{Discussion}

$\mathrm{AF}$ is a rare condition with less than 200 cases described in literature. Over the past years, only a few cases of de novo AF have been described while the widespread use of Isotretinoin has led to a greater number of cases of IIAF [6]. Nevertheless, there is not a remarkable number of data available in literature regarding the evidence- 
Fig. 3. After 3 months' ADA therapy. a Back. b Face. c Chest. ADA, adalimumab.
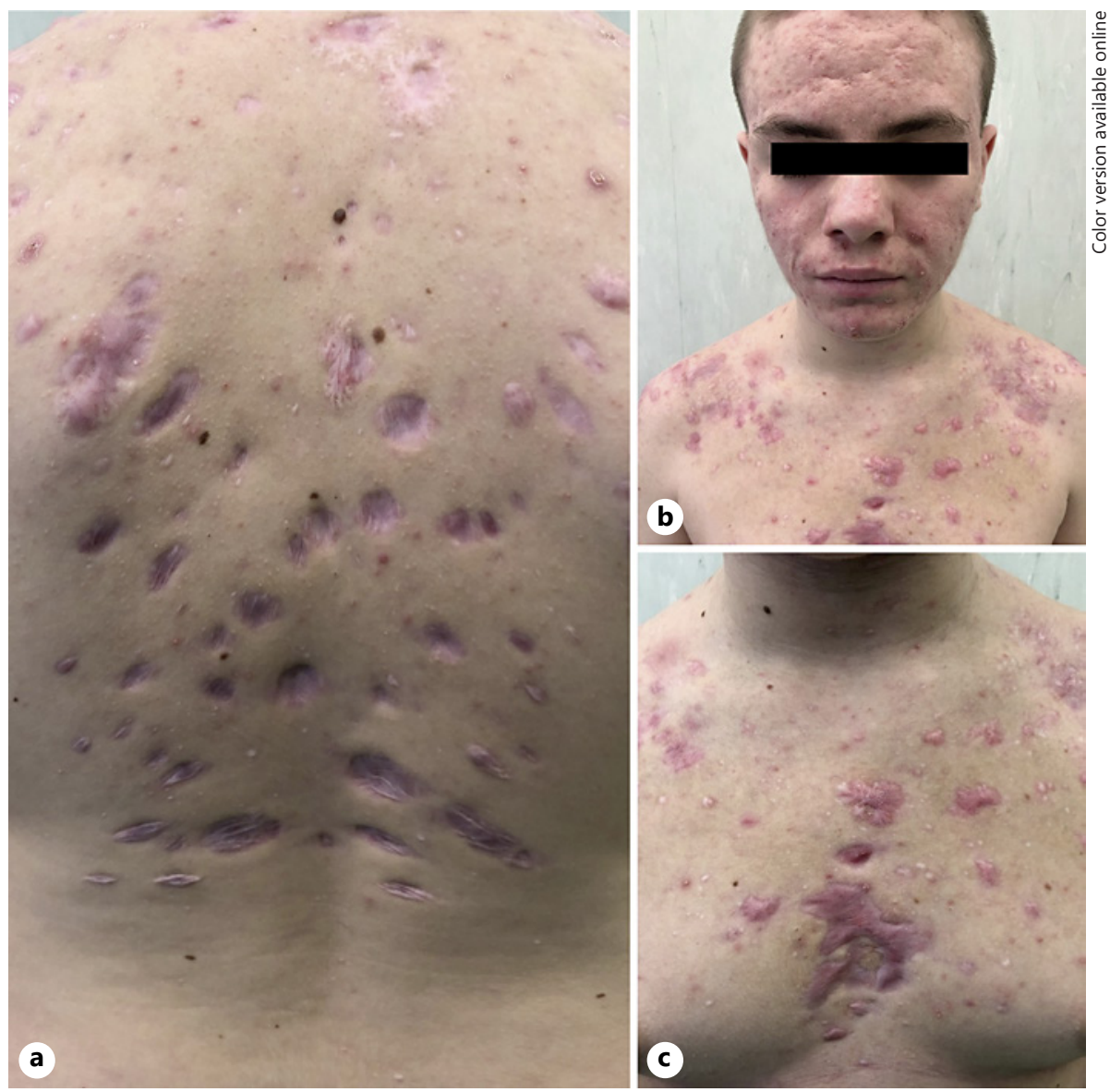

based information and no clear guidelines concerning the classification and treatment of AF.

Evidence-based recommendations for the management of AF have been recently proposed by a panel of physicians with expertise in severe acne vulgaris: they mainly pointed out the role of systemic corticosteroids, which are recommended at the immediate onset of AF to quickly control the severity of inflammation, potentially in conjunction with a reintroduction of isotretinoin therapy. Anyway, such algorithm is not successful in all patients.

Several authors have studied acne pathogenesis, particularly mTORC1 signaling: it may be involved in the proliferation of acroinfundibular keratinocytes and increased lipid biosynthesis in sebaceous glands. In addition, a greater mTOR gene expression in patients with acne compared to healthy patients has been demonstrated [7]. Moreover, the pathogenesis hypothesized for the IIAF is excessive types III and IV hypersensitivity reaction to the antigens of Propionibacterium acnes.
Isotretinoin may cause an increased fragility of pilosebaceous duct and the apoptosis of sebocytes, which lead to a massive release of pro-inflammatory cytokines in the dermis, including TNF- $\alpha$ and IL-1 $\beta[6]$. According to the algorithm by Greywal et al. [6], since our patient experienced a persistent clinical flare after reintroduction of Isotretinoin, we considered an alternative and off-label therapy with ADA.

ADA, an anti-TNF- $\alpha$ monoclonal antibody, has been approved for the treatment of multiple pediatric conditions [8]. Moreover, the efficacy of off-label therapy with ADA in the treatment of several dermatological disorders, including pyoderma gangrenosum, and IIAF has been reported [9-11]. In a previous study, it has been demonstrated that mTOR gene expression was reduced by $\mathrm{ADA}$, suggesting the tight relationship between mTOR pathway and TNF- $\alpha$ [12].

Our case has shown resolution of inflammatory skin lesions within 3 weeks, without any adverse events. An important concern about IIAF is the psychological impli- 
cation in the patient's life. AF is characterized by disfiguring lesions that could negatively impact on social activities, with a decline in the quality of life [13]. In order to improve the self-confidence of our patient, we have also proposed psychological counseling and camouflage sessions, with a significant improvement of DLQI (from 24, measured before starting treatment, to 10 , measured at week 12).

\section{Conclusion}

The aim of this case report is to improve current knowledge concerning the therapeutic management of refractory IIAF. ADA is an effective off-label option in the treatment of resistant IIAF in children. Unlike systemic corticosteroids, biological therapy does not affect bone growth and psychological status. A multidisciplinary approach is indispensable in the management of IIAF, especially in younger patients, because of psychological and aesthetic implications that may widely affect their quality of life.

\section{References}

1 Zaba R, Schwartz R, Jarmuda S, CzarneckaOperacz M, Silny W. Acne fulminans: explosive systemic form of acne. J Eur Acad Dermatol Venereol. 2011;25(5):501-7.

2 Seukeran DC, Cunliffe WJ. The treatment of acne fulminans: a review of 25 cases. Br J Dermatol. 1999;141(2):307-9.

3 Karvonen SL. Acne fulminans: report of clinical findings and treatment of twenty-four patients. J Am Acad Dermatol. 1993;28(4):5729.

4 Proença NG. Acne fulminans. An Bras Dermatol. 2017;92(5 Suppl 1):8-10.

5 Massa AF, Burmeister L, Bass D, Zouboulis CC. Acne fulminans: treatment experience from 26 patients. Dermatology. 2017;233(23):136-40.

6 Greywal T, Zaenglein AL, Baldwin HE, Bhatia N, Chernoff KA, Del Rosso JQ, et al. Evidence-based recommendations for the management of acne fulminans and its variants. J Am Acad Dermatol. 2017;77(1):109-17.

\section{Statement of Ethics}

A written informed consent was obtained from the parents of the patient for the publication of this case report and accompanying images. The authors have no ethical conflicts to disclose. The research was conducted ethically in accordance with the World Medical Association Declaration of Helsinki.

\section{Conflict of Interest Statement}

The authors have no conflicts of interest to declare.

\section{Funding Sources}

The authors declare they had no funding sources supporting this work.

\section{Author Contributions}

Claudio Marasca conceived the work. Gabriella Fabbrocini approved the version to be published. Luisa Abategiovanni wrote the manuscript and acquired data. Elisa Camela wrote the manuscript and revised it critically. Mariateresa Nocerino and Adriana Di Guida wrote the manuscript and analyzed data. Gaia De Fata Salvatores drafted the work and ensured the accuracy of any part of it.
7 Monfrecola G, Lembo S, Caiazzo G, De Vita V, Di Caprio R, Balato A, et al. Mechanistic target of rapamycin (mTOR) expression is increased in acne patients' skin. Exp Dermatol. 2016;25(2):153-5.

8 Horneff G, Seyger MMB, Arikan D, Kalabic J, Anderson JK, Lazar A, et al. Safety of adalimumab in pediatric patients with polyarticular juvenile idiopathic arthritis, enthesitis-related arthritis, psoriasis, and Crohn's disease. J Pediatr. 2018;201:166-e3.

9 Marasca C, Fontanella G, Annunziata MC, Marasca D, Fabbrocini G. Adalimumab plus topical tacrolimus for the treatment of pyoderma gangrenosum: report of a case. Int Wound J. 2019;16(4):1047-8.
10 Dawoud NM, Elnady BM, Elkhouly T, Yosef A. Adalimumab as a successful treatment for acne fulminans and bilateral acute sacroiliitis with hip synovitis complicating isotretinoin therapy. Indian J Dermatol Venereol Leprol. 2018 Jan-Feb;84(1):104-7.

11 Miguel D, Tittelbach J, Elsner P. A dramatic case of acne fulminans responding to adalimumab. J Dtsch Dermatol Ges. 2019;17(8): 837-8.

12 Balato A, Caiazzo G, Annunziata MC, Marasca C, Scala E, Cacciapuoti S, et al. AntiTNF- $\alpha$ therapy modulates mTORC1 signalling in hidradenitis suppurativa. J Eur Acad Dermatol Venereol. 2019;33(1):e43-5.

13 Zito PM, Badri T. Acne fulminans. [Updated 2020 Apr 14]. In. StatPearls [Internet]. Treasure Island, FL: StatPearls Publishing; 2020 Jan. https://www.ncbi.nlm.nih.gov/books/ NBK459326/. 